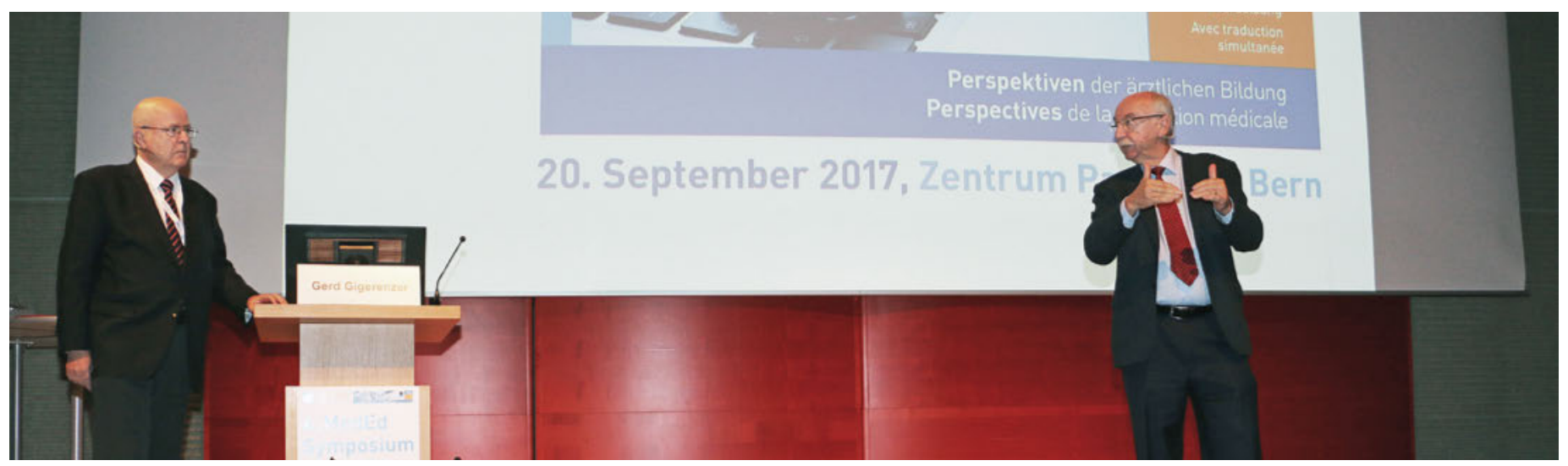

Das 4. MedEd Symposium bot Gelegenheit für einen intensiven Austausch zu den Perspektiven der ärztlichen Bildung: Gastgeber Werner Bauer (links) im Dialog mit Referent Gerd Gigerenzer.

\title{
Neue Technologien, neue Kompetenzen, neue Methoden
}

\section{Bruno Kesseli}

Dr. med. et lic. phil., Chefredaktor

In der 4. Auflage fand das MedEd Symposium des Schweizerischen Instituts für ärztliche Weiter- und Fortbildung SIWF erstmals im Zentrum Paul Klee statt. Konstant blieb die hohe Programmqualität: Spannende und informative Referate, vertiefende Seminare, ein Interview und eine abschliessende Podiumsdiskussion führten zu angeregten Diskussionen und vermittelten den Teilnehmenden wertvolle Denkanstösse.

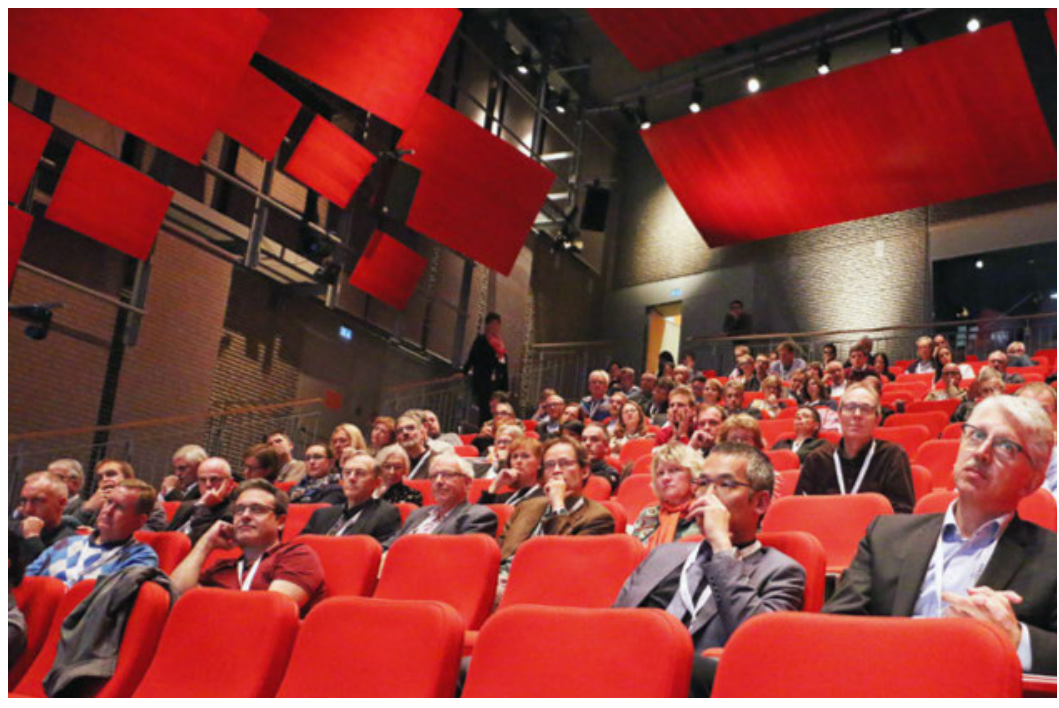

Interessiert und diskussionsfreudig: das Publikum am MedEd Symposium.
Von der Unsicherheit, unter der Ärztinnen und Ärzte häufig ihre Entscheide zu fällen haben, war am Symposium öfter die Rede. Eine Unsicherheit wurde indessen gleich zum Auftakt der Veranstaltung ausgeräumt. Die Podiumsgäste erlebten auf der Grossleinwand live mit, wie mit Ignazio Cassis ein Arzt in den Bundesrat gewählt wurde. Eine Rarität, wie SIWF-Präsident Werner Bauer in seiner Eröffnungsansprache festhielt: Ignazio Cassis ist erst der zweite Arzt in der Landesregierung. Die Amtszeit seines Berufskollegen Adolf Deucher, der ebenfalls der FDP angehörte und als Wegbereiter der SUVA gilt, liegt allerdings schon über ein Jahrhundert zurück. 1883 gewählt, starb der Thurgauer 1912 im Alter von 81 Jahren im Amt.

Das MedEd Symposium ist allerdings nicht politischen, sondern den «Perspektiven der ärztlichen Bildung» gewidmet. Es entspricht der Philosophie und dem An- 
spruch des SIWF unter der Führung von Werner Bauer, die ärztliche Bildung nicht nur zu verwalten, sondern mitzugestalten und aktiv zu deren Verbesserung beizutragen. In diesem Konzept spielt das MedEd Symposium als Austauschforum für Fachleute der ärztlichen Bildung eine wichtige Rolle. Das Erkennen und Analysieren von Entwicklungen, die Konsequenzen für die ärztliche Bildung haben, ist zentraler Bestandteil der Veranstaltung. Wie Werner Bauer festhielt, wurde für die 4. Auflage besonderes Gewicht auf neue Technologien, neue Lernziele und Kompetenzen sowie damit verbunden neue didaktische Methoden gelegt.

\section{Hohe Relevanz der ärztlichen Bildung}

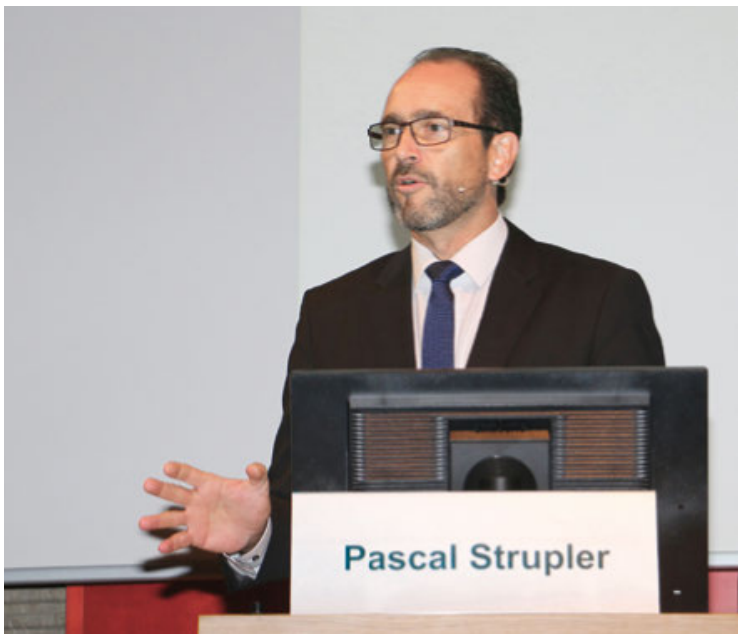

"Gehe dorthin, wo der Puck sein wird":

BAG-Direktor Pascal Strupler.

Die neuen Technologien wurden unter dem Schlagwort Digitalisierung bereits vom ersten Gastredner, Pascal Strupler, angesprochen. Der Direktor des Bundesamts für Gesundheit ist Stammgast am MedEd Symposium und eröffnete die Tagung auch in diesem Jahr mit einem Grusswort. Die Technologisierung und Digitalisierung, so Strupler, habe nicht nur Auswirkungen auf die ärztliche Bildung, sondern auf das gesamte Gesundheitswesen. Es sei deshalb sinnvoll, vertieft darüber nachzudenken, welche Erwartungen mit den aktuellen technologischen Entwicklungen verbunden seien und wohin diese uns führten. Die ärztliche Bildung, deren Relevanz für das Gesundheitswesen nicht hoch genug eingeschätzt werden könne, müsse mit genügend Vorlauf und Weitsicht gestaltet werden. Die Ärzteschaft habe unter anderem die Möglichkeit, sich via die seit 2010 bestehende BAG-Plattform «Zukunft ärztliche Bildung» in die Diskussion einzubringen. Er schloss mit einem Zitat des «begnadeten Eishockeyspielers» Wayne Gretzky: "Gehe nicht dorthin, wo der Puck ist, gehe dorthin, wo der Puck sein wird.»

\section{Informatik auf dem Vormarsch}

Überholt die Informatik die ärztliche (Fort-)Bildung? Unter diese Frage stellte Christian Lovis sein Referat [1]. Was die Lockerheit seines rhetorisch unterhaltsamen und witzigen Auftritts betraf, reihte sich der Professor für Klinische Informatik an der Universität Genf und Direktor der Division of medical information sciences des Genfer Universitätsspitals in die angelsächsische Tradition ein, wie sie von Grössen wie Steve Jobs oder Bill Gates repräsentiert wird. «Wir haben von einer langsamen in eine schnelle Zeit gewechselt», stellte er fest. Dieser Schnitt sei nicht rückgängig zu machen. Er konstatierte einen Vormarsch der Maschinen, mit denen der Mensch in Bezug auf die Analyse grosser Datenvolumen nicht mithalten kann. So integriert das von IBM entwickelte Computerprogramm Watson beispielsweise die Daten von 30 Milliarden Röntgenbildern, eine für menschliches Ermessen unvorstellbar grosse Menge an Information. Mediziner, stellte Lovis fest, würden zu wenig gut oder überhaupt nicht ausgebildet in Bezug auf künstliche Intelligenz und Big Data. Dies wäre aber vordringlich, denn die Patienten würden die Ärztinnen und Ärzte zunehmend herausfordern. Lovis verwies in diesem Zusammenhang auf Autoren wie den Amerikaner Eric Topol, der in Büchern wie «The Patient will see you now» oder "The Creative Destruction of Medicine» das revolutionäre Potential einer digitalisierten Medizin beschreibt. Ärzte sollten darauf vorbereitet werden, dass medizinische Information nicht länger nur einer Minderheit zugänglich sein wird. «Die Informatik kann helfen», bilanzierte er, aber er liess auch keinen Zweifel daran offen, dass sie in der ärztlichen Bildung einen höheren Stellenwert erhalten muss als bisher.

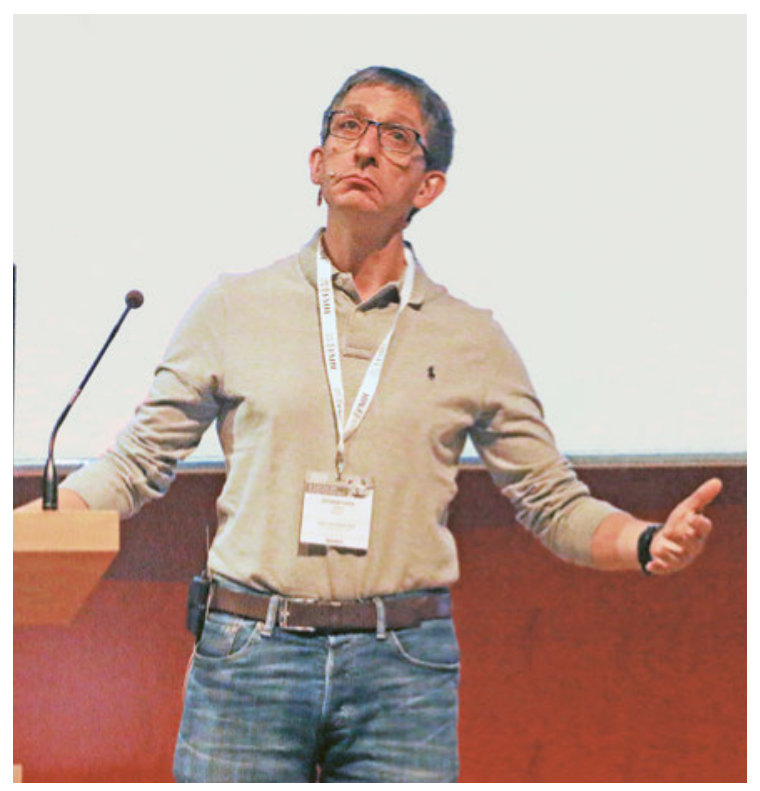

Angelsächsische Lockerheit: Christian Lovis. 


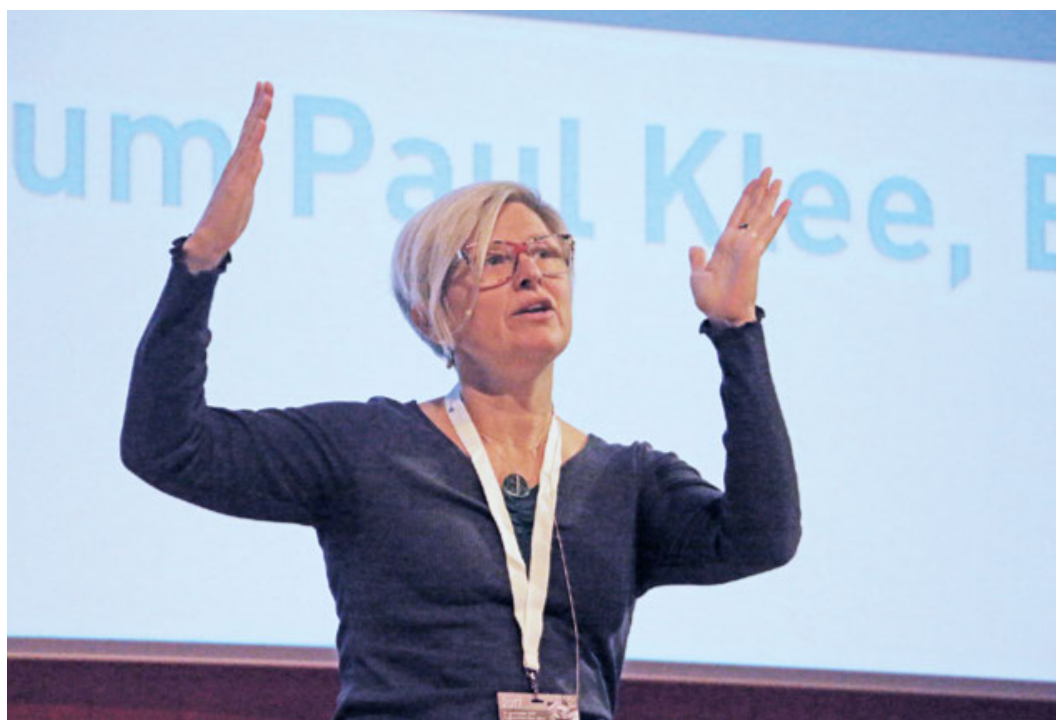

Kommunikation als ärztliche Grundkompetenz: Tanja Krones.

\section{Simulationspatienten und Blended- Learning-Konzepte}

Zeichnete Christian Lovis eher die grossen Linien der Entwicklungen im Bereich der Informationstechnologie nach, so konzentrierte Tanja Krones ihre Ausführungen auf den Nutzen konkreter Methoden in der ärztlichen Weiter- und Fortbildung. Die Professorin und Leitende Ärztin für Klinische Ethik am Institut für Biomedizinische Ethik und Medizingeschichte der Universität Zürich richtete den Fokus auf kommunikative Fertigkeiten, interprofessionelle Teamprozesse und erfolgreichen Wissens- und Skilltransfer. Kommunikation, betonte sie, sei eine zentrale ärztliche Grundkompetenz, die nicht naturgegeben sei, sondern erworben werden müsse und ein Leben lang verbessert werden könne. Gelernt würden kommunikative Fertigkeiten am besten in kliniknahen Szenarien und mit professionellem Feedback, wobei das Augenmerk auch auf interprofessionelle Teamprozesse gerichtet werden sollte. Simulationspatienten und -szenarien sind aus ihrer Sicht eine Schlüsselmethodik für den Erwerb kommunikativer Kompetenzen. Die immer wieder geäusserte Kritik an den «unechten» Simulationspatienten hält sie für ungerechtfertigt. Die Kritiker, zeigte sie sich überzeugt, hätten wohl nie ein Simulationstraining erlebt. E-Learning ist für Krones ein nützliches Instrument des Wissens- und Skilltransfers. Dies gilt insbesondere dann, wenn e-Learning im Rahmen kombinierter Konzepte wie Blended Learning oder Flipped classroom eingesetzt wird. Allerdings sollten die neuen Instrumente nicht als Ersatz der traditionellen Bildungsformen, sondern als eine zusätzliche Lernoption gesehen werden. Krones illustrierte die präsentierten Erkenntnisse an zahlreichen Beispielen aus der Praxis des UniversitätsSpitals Zürich. Die angeregte Diskussion am Ende ihres Referats zeigte, dass ihre Ausführungen beim Publikum auf grosses Interesse stiessen.

\section{«Riesige blinde Flecken»}

Wie man mit biostatistischen Daten Verwirrung stiften und manipulieren, aber auch Transparenz und Verständnis schaffen kann, demonstrierte Gerd Gigerenzer in seinem Vortrag zum Thema «Entscheiden unter Ungewissheit» eindrücklich. Der Direktor am Max-Planck-Institut für Bildungsforschung und des Harding-Zentrums für Risikokompetenz, Berlin, hat sich weltweit einen Namen als Experte auf dem Gebiet der Risikokompetenz gemacht. Die Fähigkeit, Risiken richtig einschätzen zu können, hält er in unserer Zeit für genauso wichtig wie Lesen und Schreiben vor 150 Jahren. Im Bereich der Medizin ist eine realistische Einschätzung von Risiken unter anderem auch deshalb nicht einfach, weil bei der Darstellung von Studiendaten oft getrickst wird, um die Resultate im gewünschten Licht erscheinen zu lassen. Dahinter stecken meist handfeste Interessen. Gigerenzer zeigte den Unterschied zwischen irreführender und transparenter Risikokommunikation anhand konkreter Beispiele, in denen es um Überlebensraten, relative Risiken und bedingte Wahrscheinlichkeiten ging. Er demonstrierte mit verblüffender Klarheit, wie etwa im Rahmen von Screening-Programmen mit 5-Jahres-Überlebensraten, Überdiagnosen und relativer Risikoreduktion Schindluder getrieben wird. Die Fokussierung auf relative Risikoreduktion ist auch bei der Medikamentenwerbung beliebt. So wirkt es ziemlich überzeugend, wenn ein Lipidsenker das Risiko, einen Schlaganfall zu erleiden,

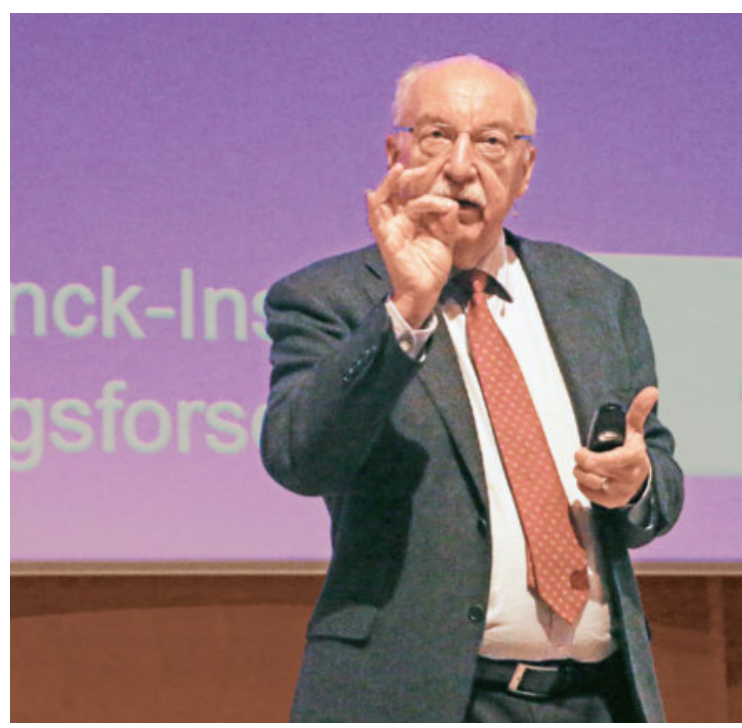

«Denken ist kein Soft Skill»: Gerd Gigerenzer. 
um 48 Prozent senkt. Im Klartext bedeutete dies im konkreten Fall eine Reduktion von 2,8 auf 1,5 Fälle pro 100 Patienten, was deutlich weniger eindrücklich ist. Wie Gigerenzer zeigte, sind leider gerade bei der Ärzteschaft in Bezug auf die Fähigkeit, biostatistische Daten zu analysieren, «riesige blinde Flecken» auszumachen. Viele Ärztinnen und Ärzte lassen sich deshalb relativ leicht hinters Licht führen. In diesem Bereich weist die ärztliche (Aus-)Bildung ein grosses Verbesserungspotential auf. Risikokompetenz ist für Gigerenzer ein zentraler Teil der digitalen Revolution. Grundsätzlich sei es eine wichtige Aufgabe, den Medizinstudenten «Denken» beizubringen, zeigte er sich überzeugt: «Denken ist kein Soft Skill.»

\section{Drei Workshops und ein Interview}

Traditionellerweise wurde der Symposiumsnachmittag durch drei parallel geführte Workshops [1] eingeleitet, in denen nach Inputreferaten interaktiv die folgenden Themen behandelt und diskutiert wurden:

- Künstliche Intelligenz und ärztliche Bildung: Wohin führt der Weg? [2]

- Neue Methoden in der Weiterbildung: Nice to have or need to have? [3]

- Médecin et après? Les enjeux du choix de carrière. [4]

Danach standen die Ärztinnen und Ärzte in Weiterbildung für eine halbe Stunde nicht nur theoretisch, sondern in Fleisch und Blut im Mittelpunkt. Werner Bauer bat Livia Dülli und Rolf Erlebach zum Interview auf die Bühne. Beide arbeiten zurzeit als Assistenzärzte am Kantonsspital Winterthur, Livia Dülli in der Pädiatrie, Rolf Erlebach in der Anästhesie. Beide erleben den Stellenwert der Weiterbildung in ihren Abteilungen grundsätzlich als hoch, das Klima als weiterbildungsfreundlich,

\section{SIWF-Award 2017: die Ausgezeichneten}

Im Rahmen des 3. MedEd Symposiums wurden auch die Trägerinnen und Träger des SIWF-Awards 2017 für besonderes Engagement in derWeiterbildung bekanntgegeben. Diese Auszeichnung wird aufgrund der Nomination durch ehemalige Assistenzärztinnen und -ärzte vergeben. Folgende Weiterbildner erhielten in diesem Jahr eine Auszeichnung:

Herr Dr. med. Frank Bochmann, Luzern; Frau Dr. med. Johanna Büchel, Basel; Monsieur le Docteur Christian Candrian, Lugano; Herr PD Dr. med. Heiko Frühauf, Zürich; Herr Dr. med. Andreas Geiser, Schlieren; Frau Dr. med. Andrea Grêt, Solothurn; Herr Prof. Dr. med. Andreas Günthert, Luzern; Frau med. pract. Ann-Kristin Hörsting, Winterthur; Monsieur le Docteur Lionel Jacquier, Martigny; Madame la Doctoresse Rachida Marir, Lausanne; Frau Dr. med. Luzia Meier, Basel; Herr Prof. Dr. med. Dr. sc. nat. Alexander A. Navarini, Zürich; Frau PD Dr. med. Cäcilia Reiner, Zürich; Monsieur le Docteur Diego San Millán, Sion; Herr Dr. med. Thomas Sauter, Bern; Herr PD Dr. med. Parham Sendi, Basel; Monsieur le Docteur Christophe Sénéchaud, Saint-Imier; Herr Dr. med. Philipp Stillhard, Chur; Frau Dr. med. BirgittaThomann, Schlieren; Herr Prof. Dr. med. Stephan Vavricka, Zürich; Herr PD Dr. med. Mathias Worni, Bern.

auch wenn es punktuell an den meisten Weiterbildungsstätten heikle Bereiche gebe. Die von ihnen angeführten Beispiele waren denn auch nicht spezifisch für ihre aktuellen Arbeitsplätze, sondern betrafen Schwierigkeiten, von denen beim Austausch mit Kolleginnen und Kollegen öfter die Rede war. So hätten Weiterbildungsanliegen einen schweren Stand, wenn sie mit der vorgeschriebenen Arbeitszeit in Konflikt kämen oder in speziellen, schwierig planbaren Konstellationen wie bei Einsätzen auf der Notfallstation. Negativ sei in der täglichen Arbeit spürbar, dass die Büroarbeit zulasten der Zeit zugenommen habe, die für den direkten Kontakt mit den Patienten zur Verfügung stehe. Mehr BedsideTeaching wäre wünschenswert; generell seien Feed-

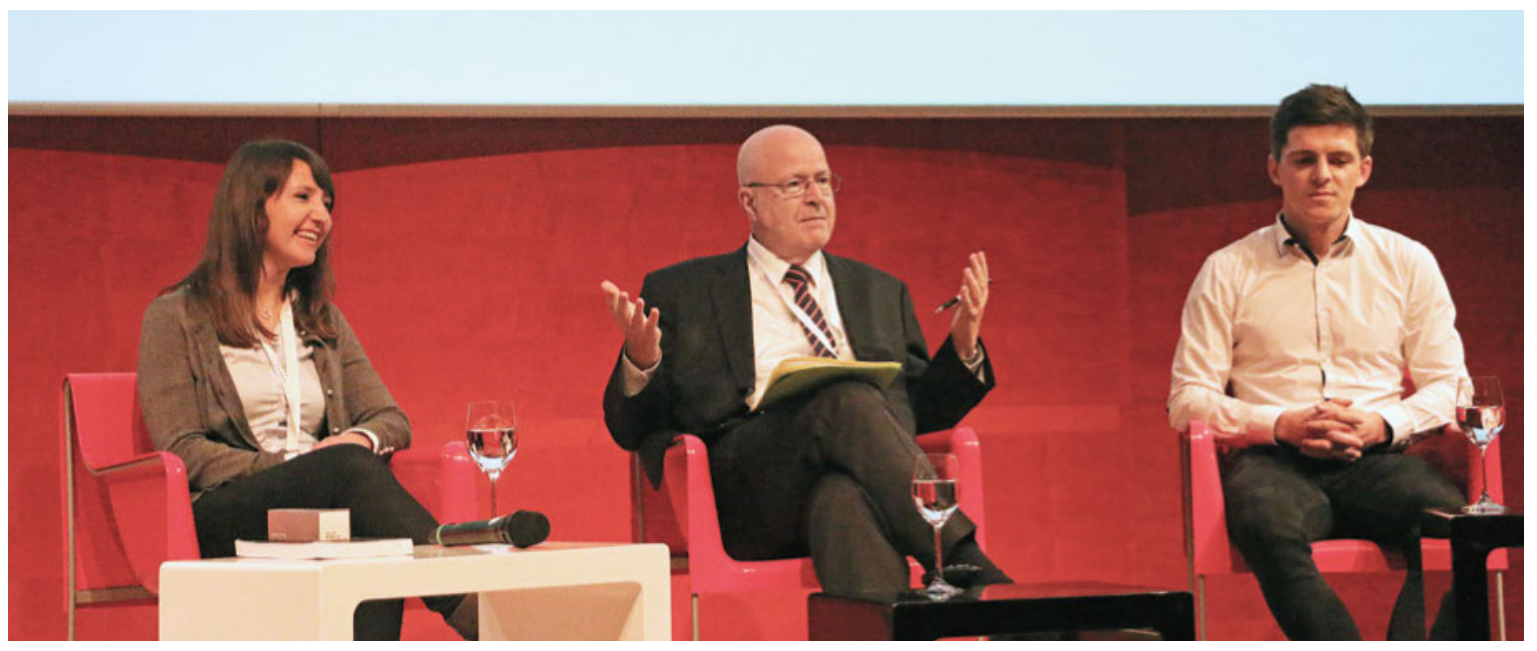

«Jetzt reden wir»: Werner Bauer im Gespräch mit Livia Dülli und Rolf Erlebach, Ärztin und Arzt in Weiterbildung. 
backs durch erfahrene Kolleginnen und Kollegen sehr wertvoll. Dass sie solche Feedbacks im Rahmen der vorgeschriebenen Arbeitsplatz-basierten Assessments viermal jährlich sicher erhalten, werteten beide Interviewten als positiv. Die jährliche Umfrage zur Evaluation der Weiterbildungsstätten sehen sie als wichtiges Instrument der Qualitätssicherung, das unter anderem für die Bewerbungen hilfreich sei und dafür auch von vielen ihrer Kolleginnen und Kollegen genutzt werde.

\section{Lebhaftes Podium}

Auf dem abschliessenden Podium schaffte es Moderator Iwan Rickenbacher, mit seinen Gästen [5] in konzentrierter Form die Kernthemen des Symposiums nochmals aufzugreifen und in den grösseren Kontext der Gesundheitspolitik zu stellen. Dabei zeigte sich unter anderem, dass Innovation von den Experten durchaus differenziert beurteilt wird. Der Stellenwert des Arzt-Patienten-Verhältnisses werde unabhängig von der Innovationsbeschleunigung hoch bleiben, zeigte sich Henri Bounameaux überzeugt. Sein Kollege Bruno Schmied betonte, auch Innovation sei kritisch zu hinterfragen. Man müsse sich gut überlegen, welche Innovationen etwas bringen würden, gerade im Weiterbildungsbereich. Interessante Statements waren zum Thema «Steuerung» oder - politisch korrekter - «Koordination der ärztlichen Weiterbildung» zu verzeichnen. Stefan Spycher wies zwar auf «Herausforderungen» hin, stellte den etablierten Weiterbildungspfad aber

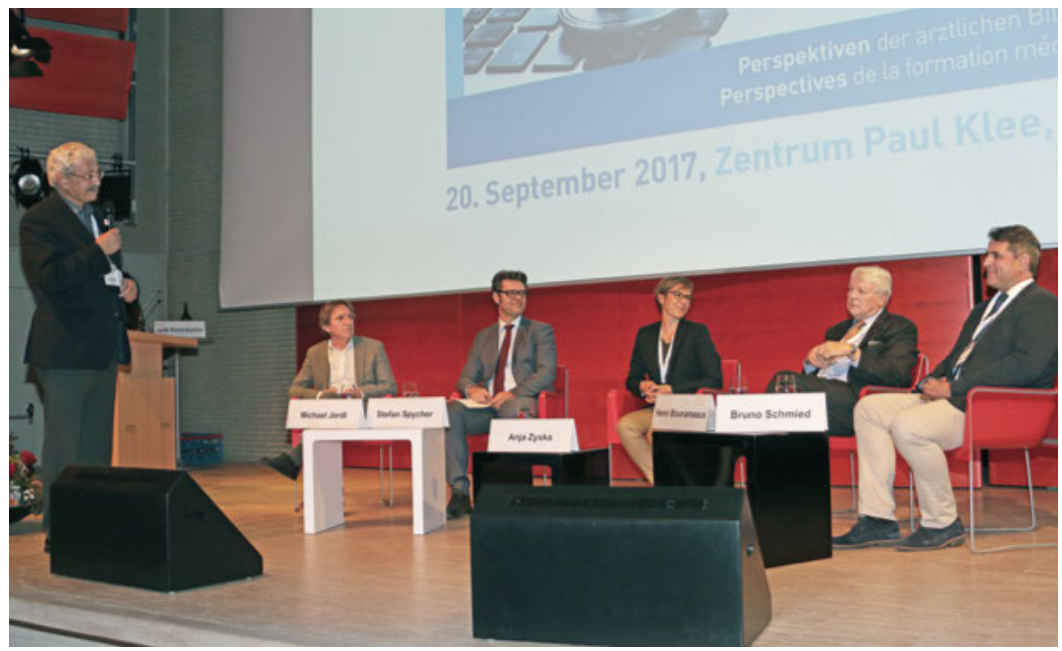

Animierte Diskussion (v.l.n.r.): Moderator Iwan Rickenbacher, Michael Jordi, Stefan Spycher, Anja Zyska, Henri Bounameaux, Bruno Schmied. nicht grundsätzlich in Frage. Henri Bounameaux fand es dagegen gerechtfertigt, über Regulierungen nachzudenken. Freiheit sei zwar gut, aber im Weiterbildungsbereich sei das Resultat nicht immer überzeugend, wenn man an das Verhältnis zwischen Grundversorgern und Spezialisten denke. Klar gegen politisch orchestrierte Steuerungsmassnahmen sprach sich Anja Zyska aus, weil dafür schlicht die Datenbasis fehle. Zudem gehe es nicht an, Ärztinnen und Ärzte aus- und weiterzubilden und ihnen dann die Zulassung zu verweigern. Auf Stufe Weiterbildung sähe sie zwar gewisse Steuerungsmöglichkeiten, aber das Problem sei die Bedarfsabschätzung. Michael Jordi outete sich als Pragmatiker, der nicht auf perfekte Zahlen warten, sondern Probleme lösen möchte. Steuerungsmöglichkeiten sehe er zum einen bei der Zulassung zum Studium. Zum andern sollten die Kantone niederschwellig eingreifen können, wenn die Situation "aus dem Ruder läuft», wie dies etwa für die Kantone Genf, Waadt, Tessin oder St. Gallen gelte. Dem abschliessenden Votum von Anja Zyska konnten sich vermutlich die Podiumsteilnehmer wie auch der Grossteil des Publikums anschliessen. Eine qualitativ hochwertige Weiterbildung mit definierten Stundenzahlen, wie wir sie in der Schweiz haben, gebe es in dieser Art anderswo kaum. «Das müssen wir uns bewahren.» Eine analoge Aussage über das MedEd Symposium wäre auch nicht verfehlt. Für 2018 kann man sich den Termin jedenfalls bereits vormerken: 12. September, Zentrum Paul Klee.

\section{Bildnachweis}

Fotos Bruno Kessel

\section{Anmerkungen}

1 Die Präsentationen zu den Hauptreferaten und Seminaren sind zugänglich unter:

www.fmh.ch/bildung-siwf/themen/meded-symposium/2017.html

2 Leitung: Prof. Dr. med. Giatgen A. Spinas, Pr Dr méd. Christian Lovis, Dr. Karin Vey.

3 Leitung: Dr. med. Regula Schmid, Prof. Dr. med., Dipl. Soz. Tanja Krones, Dr. med. Lukas Hegi.

4 Leitung: Dre Nadia Bajwa, Dre Anja Zyska, Dre Milena Abbiati, Dre Nathalie Koch.

5 Auf dem Podium diskutierten: Prof. Dr. Iwan Rickenbacher (Moderation), Honorarprofessor für politische Kommunikation der Universität Bern; Prof. Dr. med. Henri Bounameaux, Dekan der medizinischen Fakultät der Universität Genf, Präsident des Collège des Doyens, Direktor Ausbildung und Forschung, Hôpitaux Universitaires de Genève (HUG), Genf; Lic. rer. pol. Michael Jordi, Zentralsekretär der Schweizerischen Gesundheitsdirektorenkonferenz (GDK), Bern; Prof. Dr. med. Bruno Schmied, Chefarzt Klinik für Allgemein-, Viszeral-, Endokrin- und Transplantationschirurgie, St. Gallen; Dr. Stefan Spycher, Vizedirektor, Leiter Direktionsbereich Gesundheitspolitik, Bundesamt für Gesundheit (BAG), Bern; Dr. med. Anja Zyska, Vizepräsidentin, Verband Assistenzund Oberärztinnen und -ärzte (VSAO/ASMAC), Bern. 